\title{
CATARACT SURGERY*
}

\author{
BY
}

JOAQUIN BARRAQUER and JØRN BOBERG-ANS

From the Clinica Barraquer, Barcelona (Chief: Prof. Ignacio Barraquer) and the Finsen Institute, Eye Department (Chief: Dr. O. Blegvad)

EXTRACTION of the opaque intra-ocular lens has always been a favourite and important eye operation. The dexterity and the instruments needed for the removal of the cataractous lens have attracted the interest of ophthalmologists and have called forth a vast literature on the various methods of miraculously restoring the power of sight to the blind.

Operating Chair.-One of the fundamental conditions for a successful operation is the dexterity and steadiness of the surgeon, and it is essential that his hands and forearms be supported while he works.

The surgeon should be seated at the head of the operating table. He should sit comfortably, with a good back support, and his elbows and forearms should be supported by an arm rest, firmly fixed to the chair. This arm rest should be covered with a sterile cloth. The patient should be placed so that his eye is in a convenient working position for the surgeon's hands, and also within normal working distance from the surgeon's eyes. The surgeon should not have to bend his head or neck awkwardly in order to see well.

At the Barraquer Clinic, a special chair is used, with arm and back rests formed in one piece. Below this is another support for the assistant's arm, should it be necessary for him to cross the arm of the surgeon. The upper support is thus interposed between the arm of the assistant and that of the surgeon and, in this way, the one is prevented from disturbing the other's movements (Fig. 1a).

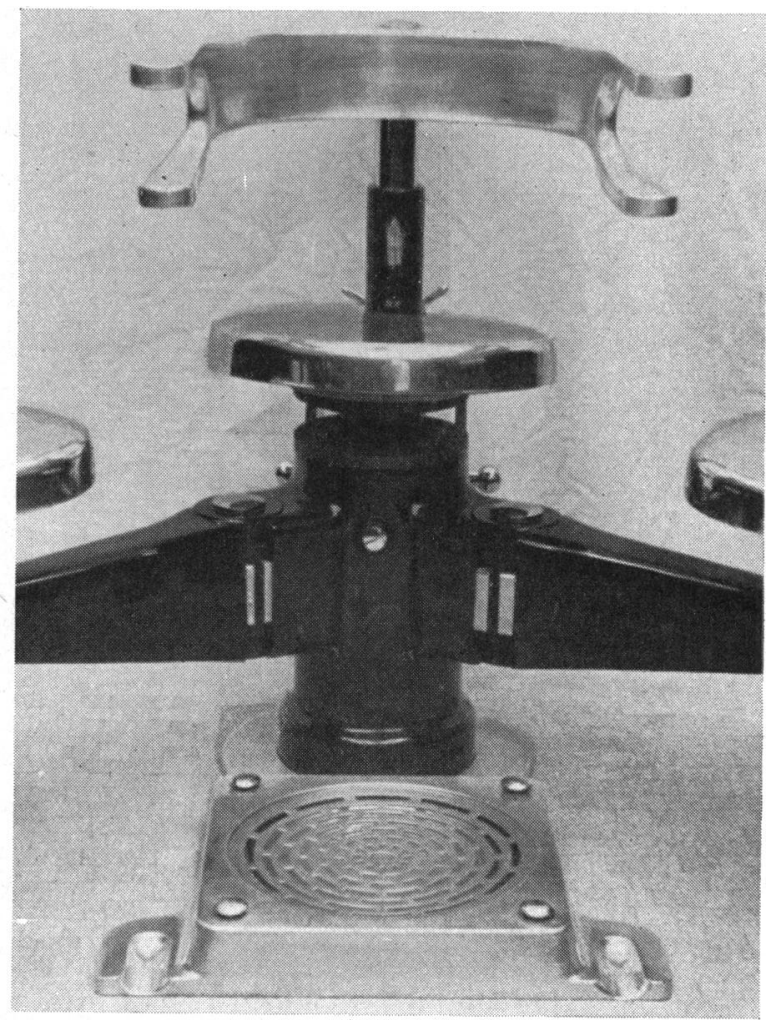

FIG. 1a.-Barraquer operating chair, with arm rests for the surgeon and the assistants, all made in one piece. There are seats for both surgeon and assistants, and a foot rest which includes spaces to receive the wheels of the stretcher-table.

* Received for publication November 5, 1957. 
An operating chair which conforms with these requirements, but with some modifications and without an arm rest for the assistant is used at the Finsen Institute* (Fig. 1b).

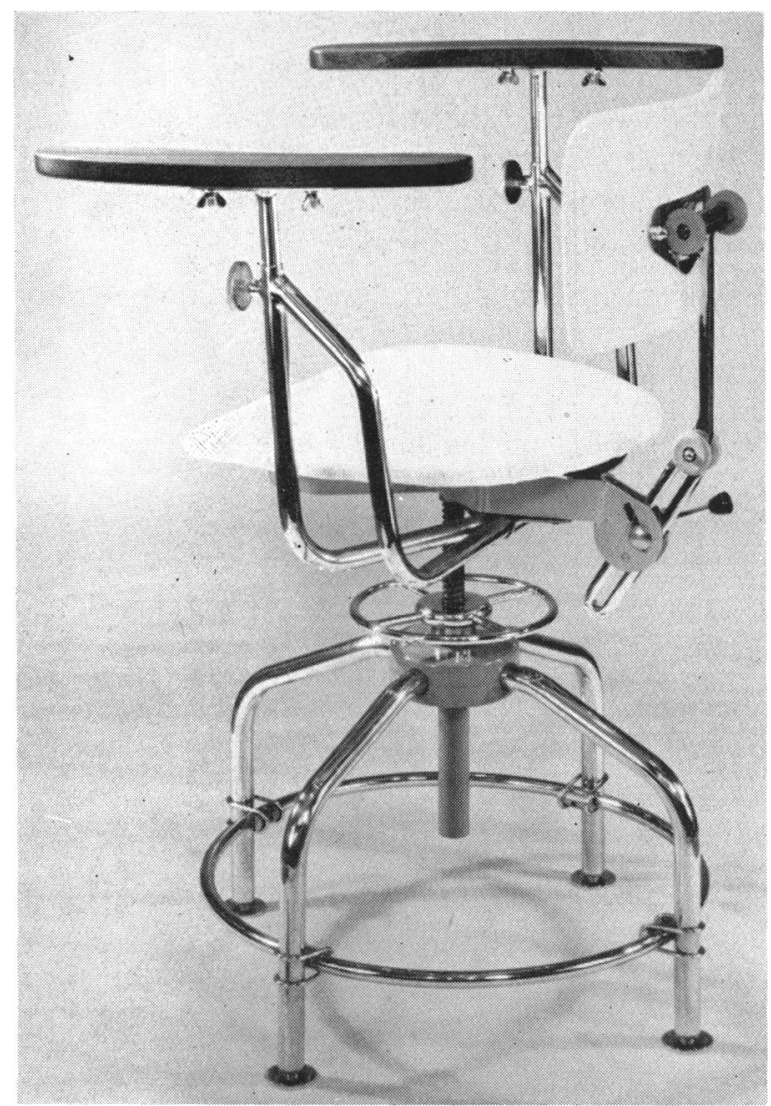

Fig. 1b.-Boberg-Ans operating chair with arm rests which are adjustable in height, projection, and angle. It is convenient that the support for the arm working from the nasal side should be higher, project further forward; and turn further inwards.

Operating Table.-This should be constructed with an adjustable head rest, so that the patient's head can be placed above the knees of the surgeon and kept quite still. A mechanically adjustable head rest, which supports and stabilizes the head, is in use at the Finsen Institute.

Lighting.-This should be uniform and not too strong, so that the patient can be kept as quiet and calm as possible. The light in the operating field should be strong enough to show up the smallest details but not so strong as to annoy the patient or dazzle his other eye.

An ordinary operating lamp gives a very strong light with a rather large field covering the face of the patient. Barraquer, therefore, uses a headlamp with a yellow bulb. A headband carries a horizontally placed reflector under which the bulb is held shaded against the surgeon's eyes (Fig. 2a, opposite).

A similar lamp (Castanera) has been found completely satisfactory at the Finsen Eye Department (Fig. 2b, opposite).

The lamp may be worn for several hours without fatigue and may become quite warm without discomfort to patient or surgeon. It permits the wearing of glasses or of a loupe and presents no obstacle to the use of an operating microscope.

* Kevi A/S, Copenhagen. 


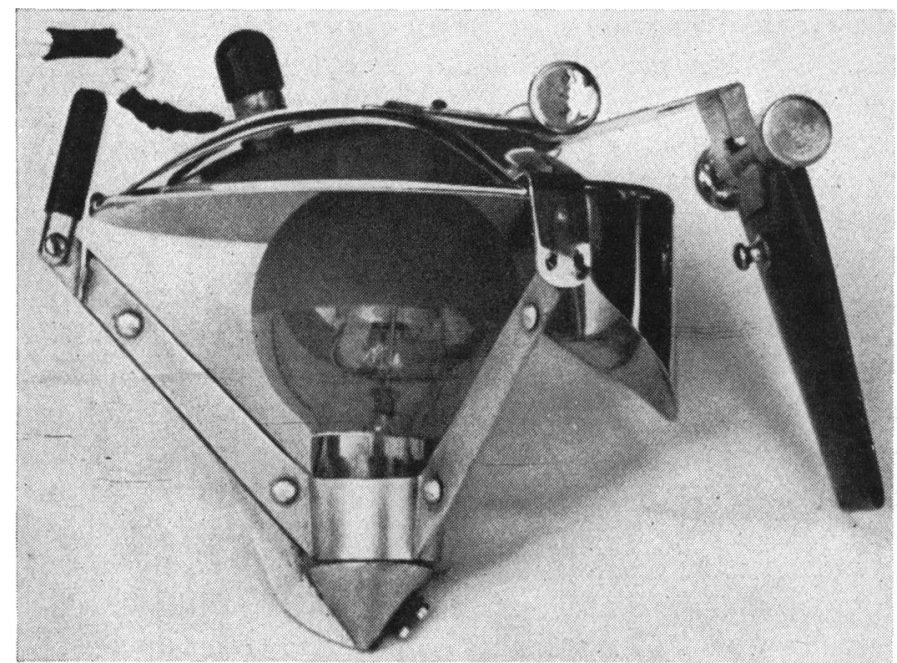

Fig. 2a.-Barraquer headlamp (Drapier, Paris).

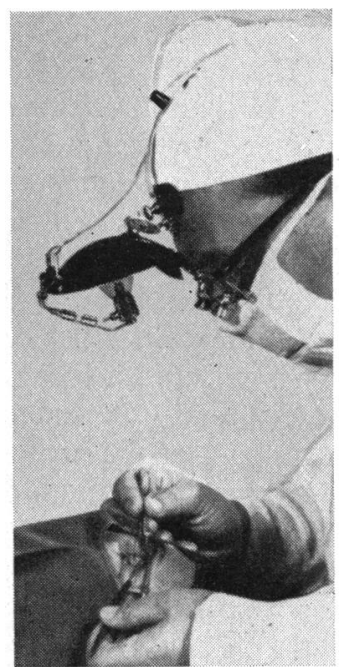

FIG. 2b.-Castanera headlamp (Casa del Medico, Barcelona).

\section{Anaesthesia}

The state of the patient during the operation is of the greatest importance. It is usually necessary to have some sort of sedation before the operation, but total anaesthesia entails inevitable moments of danger through uncontrolled movements of the patient, and the essential tranquillity and quietness can be achieved by other less risky means (Barraquer, 1954; Atkinson, 1955).

The Barraquer Clinic advocates a sedation starting the evening before the operation, supplemented on the morning of the operation, and enhanced just before the operation with pethidine and curare. Local anaesthesia of the cornea is further obtained with 2 per cent. tetracaine solution and 4 per cent. cocaine solution instilled into the eyes.

Curare gives a general relaxation acting first on the peri-ocular muscles, and causing a decrease of venous tension and depletion of the orbital veins. If the anterior chamber of the eye is opened in a non-curarized patient, the lens will move a little forward and the pupil will contract a little. If, however, the patient has received curare the lens will move backwards, leaving a slightly dilated pupil and an unsupported iris (Fig. 3). This effect does not occur even with a perfect retrobulbar anaesthesia with hyaluronidase added to the anaesthetic solution.

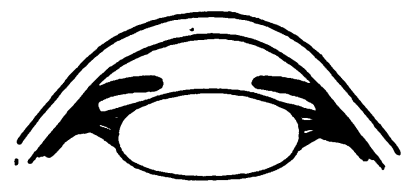

I

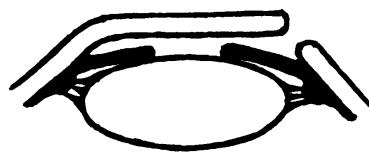

2

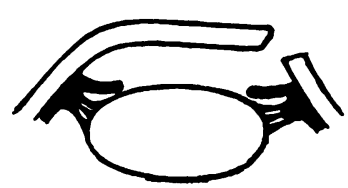

3

Fig. 3.-Anterior chamber: (1) Before operation; (2) Opened in a non-curarized patient; (3) Opened in a curarized patient, showing dilation of pupil and retraction of lens. 
At the Finsen Institute, Dr. E. Nandrup of the Department of Anaesthetics (Head: Dr. H. Ruben) has supervised the sedation and anaesthesia for our cases.

In the majority of our Danish patients at the Finsen Institute we have not reinforced the sedation with curare, but have used phenergan, barbiturates, chlorpromazine, and pethidine, with immediate pre-operative akinesia of the lids and local and retrobulbar anaesthesia.

Local anaesthesia of the cornea and conjunctiva with drops of 1 per cent. tetracaine solution is supplemented by a percutaneous retrobulbar injection of $1.5 \mathrm{ml}$. of a 2 per cent. xylocain-adrenalin solution, with hyaluronidase $2.5 \mathrm{VRE}$ per $\mathrm{ml}$. and a local anaesthesia of the conjunctiva at the superior rectus muscle. Akinesia (O'Brien, modified by Atkinson), with $2 \mathrm{ml}$. of the anaesthetic solution in front of the ear, gives the patient a painless operation with no untoward effects. Atkinson, basing his findings on a very wide series of inquiries, emphasizes that in cases of intra-capsular surgery, no movement of the patient, intentional or otherwise, must be allowed to endanger the result of the operation.

In operations by the extra-capsular method, it may be safe to operate without sedation, as has been done for many years, and is still practised by many eye surgeons.

\section{Operative Technique}

At the Barraquer Clinic, the assistant holds the lid-retractors during the operation, making it possible to alter the width of the aperture. At the Finsen Institute, a modified Arruga blepharostat $\dagger$ with a pin for fixing the suture of the rectus superior is used, and the eye is kept in a slightly downward-looking position. In this new eyelid-holder a ring-screw is substituted for the usual nasal screw. The branch for the lower lid consists of a hollow tube with perforations in the part which rests in the lower fornix. Suction drainage secures the evacuation of fluid from the conjunctival sac during the operation, thus diminishing the risk of infection and preventing blood from being sucked into the anterior chamber (Fig. 4).

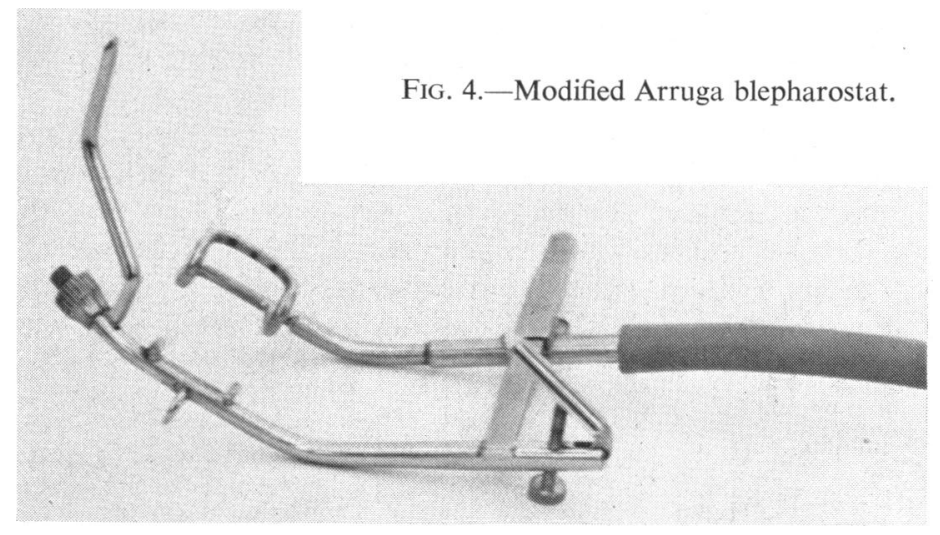

The eye is further fixed with a fixation forceps, preferably the BarraquerLloberas forceps (Fig. 5, opposite).

The incision is made with a von Graefe knife and a section of $180^{\circ}$ is made. A conjunctival flap is made upwards, the size being of little importance so long 


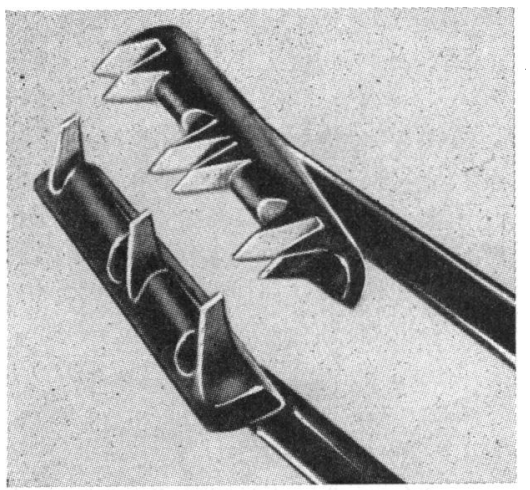

Fig. 5.-Barraquer-Lloberas fixation forceps. They have a firm grip and do not damage the conjunctiva.

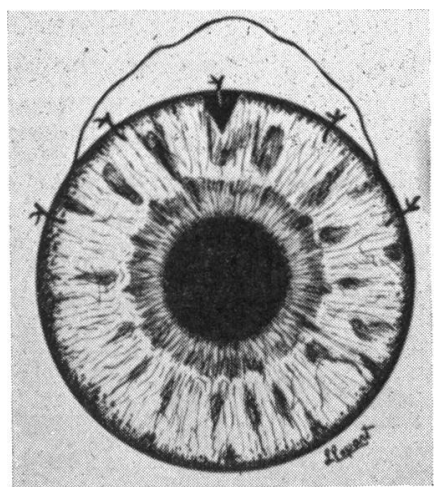

FIG. 6.-Corneo-scleral sutures with "virgin silk". The 12 o'clock suture is put in before the lens extraction. Three sutures may suffice, but five or seven are better.

as the flap is able to cover the corneo-scleral stitches, which are put in at 12 , 10.30 , and 1.30 o'clock. The two latter sutures are frequently put in after the lens has been removed (Fig. 6), and five or even seven sutures may be needed. The 12 o'clock suture is placed first. The needle is inserted first in the cornea at the edge of the wound, and it emerges in the cut just in the middle of the cut edge. It is then passed into the scleral area, care being taken to place the needle at the same level in the scleral lip as in the corneal lip of the wound (Fig. 7).

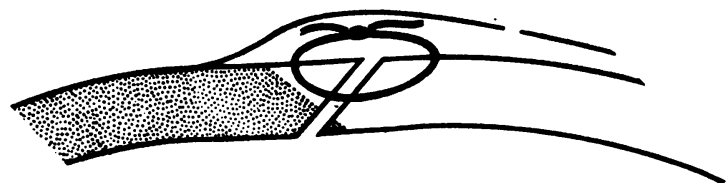

FIG. 7.--Siting of sub-conjunctival corneo-scleral sutures, showing how the needle must pierce the lips of the wound in the middle so that they can be accurately apposed.

Magnification is here indispensable. The loop of the thread is drawn out between the lips of the wound to make room for the later passage of the lens. The corneal flap is lifted by the assistant and a very small iridotomy is done at 12 o'clock. This iridotomy may be double, each cut being sited between the sutures, i.e. at 11 o'clock and at 1 o'clock.

A mohair bruṣh, sterilized by boiling for at least 15 minutes, is used to dry or suck up fluid from the wound. The brush is excellent for drying away clots either from the wound itself or from the anterior chamber, and it will cause no damage to any of the structures. When the brush is dried in a piece of cloth it is surprising to see how much fluid it has sucked up. It is useful in spreading out the conjunctival flap, re-positioning the iris, and widening the pupil. When the pupil is sufficiently large, the erisophake is introduced.

Erisophake.-The idea of Stoever (1902) and Hulen (1910) was perfected and popularized by Barraquer (1917), who made a special cup and suction apparatus for the extraction of the cataract. The construction of the cup is of importance 
to the success of the procedure (Fig. 8); it should be rather deep and the suction pipe should be placed at the edge of the cup, some way down a groove in the interior of the cup. This prevents the hole being blocked by the lens when it is sucked violently into the erisophake. With faulty construction the cup may take a firm grip of the lens in situ, but the loosened lens may block the suction pipe, with the result that the capsule ruptures or the erisophake slips off. The edge of the erisophake and the ring-shaped surface meant for contact with the lens capsule should be very even and should be shaped to fit the anterior capsule of the lens.

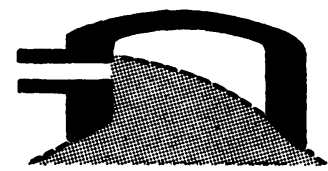

' I

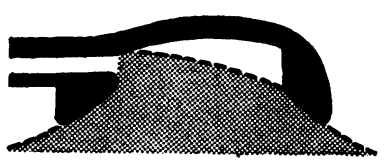

2

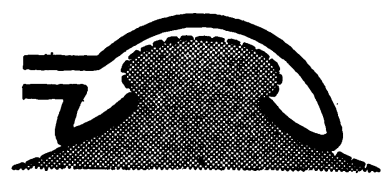

3

FIG. 8.-(1) and (2). Other types of erisophake cup.

(3) Barraquer erisophake cup, showing how the "collar" prevents blockage of the suction tube. The anterior lens capsule is sucked into the cup in a mushroom shape, which not only gives a firmer grip but also prevents any sliding on the surface. The "collar" must be free from sharp edges or points which might rupture the capsule.

Villaseca (1956) has shown that the Barraquer model has the firmest grip and will hold the heaviest weight. The "collar" prevents blockage of the vacuum tube, and the lens is sucked into the cup in a mushroom-like shape which will not slide about. The vacuum may be created by a pneumatic pump or by a rubber balloon. The latter is less reliable and the suction created may vary from 70 to $450 \mathrm{~mm}$. Hg. A mechanical pneumatic pump will be able to create a vacuum of up to $600 \mathrm{~mm}$. $\mathrm{Hg}$ and the Barraquer Clinic recommends a vacuum of 400 to $500 \mathrm{~mm}$. $\mathrm{Hg}$.

At the Finsen Institute, a Reciprotor pump and a vacuum flask have been found very satisfactory, and a vacuum of 350 to $400 \mathrm{~mm}$. Hg is used. The vacuum pump requires a valve controlled either by hand on the handle of the erisophake or by a foot pedal.* This valve controls the vacuum in the erisophake, and allows the immediate suspension of the vacuum in the cup, should this become necessary in an emergency (Fig. 9).
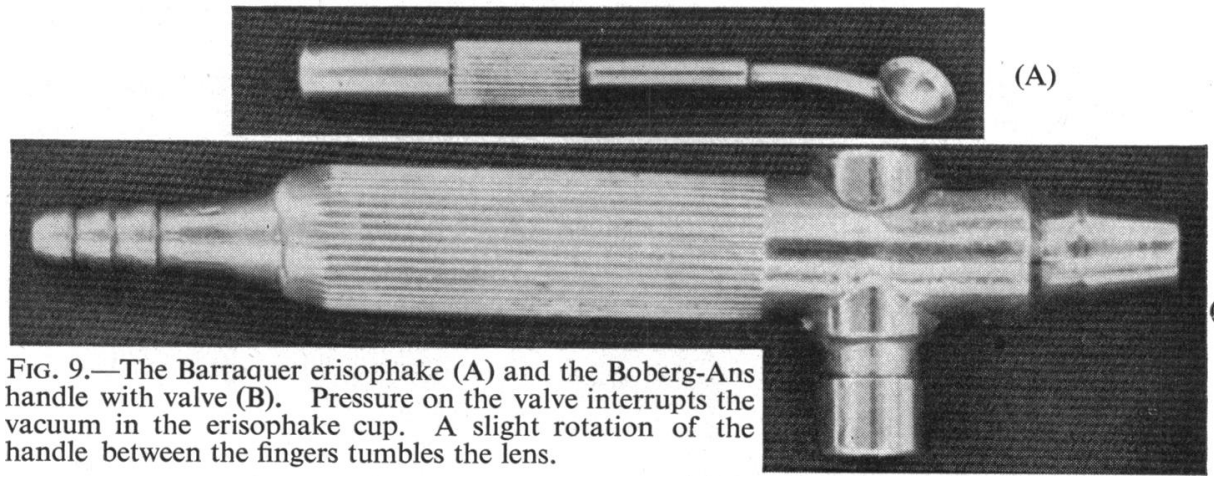

* Simonsen and Weel, Copenhagen. 
Sutures.-The needles for the sutures should be very fine: 5,7 , and $10 \mathrm{~mm}$. (Vogt-Barraquer No. 81 Grieshaber; Castroviejo No. 82 Grieshaber), or $4 \mathrm{~mm}$. in length with a more pronounced curve (Barraquer No. 83 Grieshaber). These very fine needles are best handled with a special needle-holder without a catch but with rounded handles permitting the insertion of the needles by finely controlled finger movements.

The needle is slightly curved and its concave side should be flat, with the cutting edge on the convex side. The eye of the needle is grooved on each side, thus preventing the thread from adding to the thickness of the needle. The thread is of very fine "virgin silk" (i.e. silk-worm gut filament of 1-2/2000 of an inch in its natural state: Malbrán, 1957). These are made by Ibañez, Barcelona, to the requirements of the Barraquer Clinic. The silk is dyed with a 2 per cent. solution of methylene-blue and the colour disappears soon after the insertion of the thread into the tissues. The threads do not cause any reaction whatsoever and may be left indefinitely.

Extraction of the Lens.-The cornea is lifted straight up by the assistant (as is also recommended by Kirby, 1955) in order to facilitate the sideways introduction of the erisophake, without injuring the corneal endothelium. It is better to lift the cornea fairly high so that it is not harmed by being bent over (Kirby, 1950). Striate keratitis is nearly always caused by direct injury to the posterior surface of the cornea.

The erisophake is introduced through the pupil, and is applied to the front surface of the lens as far as possible towards the lower border. It is most important to avoid any kind of pressure. When the cup is in full contact with the anterior surface of the lens, the vacuum is applied, and the erisophake takes a firm grip on the lens, which is clearly felt. A little traction in the erisophake will now lift the lens slightly forward. This forward traction is very important as it prevents any pressure on the vitreous and tightens the zonular threads. A very careful rotary movement of the erisophake and a fine steadying touch without pressure with the Colibri forceps will now break the zonular threads.* By turning the erisophake and continuing the rotation, the lens is easily tumbled out (Fig. 10), much as a button passes through a buttonhole, and, as, the forceps follow the movements, the wound is immediately closed.

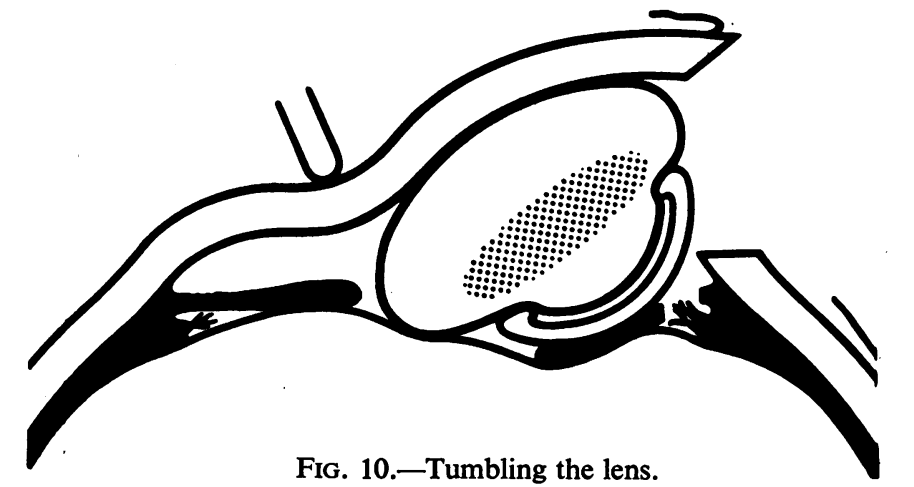

* When $\alpha$-chymotrypsin (Quimotrase, P.E.V.Y.A. Barcelona) for chemical zonulysis (Barraquer, 1958) is used, the zonular threads do not offer any resistance and no counter-pressure is required. This makes the procedure safer. $\alpha$-Chymotrypsin has also been used at the Finsen Eye Department with great success. 
The iris is replaced by means of the brush and the sutures are tied. If the sutures at 10 o'clock and 2 o'clock have not been put in, they are now inserted. The conjunctival flap is brushed into place. It is lifted carefully and plasma is deposited on the raw surface, followed by thrombin, which makes the surfaces adhere firmly together. Sutures in the conjunctiva should then be unnecessary.

\section{Discussion}

The advantages of this operative method are manifold. The sedation, anaesthesia, and curarization secure a calm and quiet patient and a soft eye with very little risk of bleeding or vitreous loss.

A clean corneal incision with a conjunctival flap helps in closing the wound more safely, and corneo-scleral sutures inserted immediately after the incision with the aid of magnification, should prevent the development of astigmatism. It is an advantage that the sutures are placed sub-conjunctivally and that their final removal is unnecessary.

Capsular forceps take hold of a small part of the lens capsule only and may easily tear it when too much pull is exerted. When the forceps are applied it is necessary to press a little on the surface of the lens, which may cause dislocation of the lens into the vitreous, or give rise to a slight prolapse of the vitreous into the wound. This will never happen with the erisophake, which takes a firm, wide grip on the anterior lens capsule, and stretches the capsule over the posterior pole of the lens, pulling the zonular threads forward, and making it easier for these to be broken (Harrington, 1951). The tumbling process makes it easier to get the cataractous lens through the pupil, even if this is not properly dilated. It is often unnecessary to re-position the iris, which is very often left undisturbed. The firm grip of the erisophake on the anterior surface of the lens makes it easy to tear even rather strong zonules. This may theoretically cause a loosening of the attachment to the ora serrata, but if one is careful to break the zonule at 6 o'clock first and the rest of it when the lens is tumbled, to lift it straight up, and to slide the lens sideways through the side of the wound, at the same time closing the wound at 12 o'clock, little effort is required to break the zonule here.

When the lens has been extracted, the anterior chamber is irrigated with a solution of acetylcholine, which contracts the pupil and prevents the vitreous from emerging. Physiological saline solution is used for further irrigation.

When the eye is closed at the end of the operation, air is injected into the anterior chamber, thereby preventing the iris and the vitreous from coming into contact with the posterior surface of the cornea.

Cortisone ointment applied at the end of the operation will shorten the period of post-operative corneal oedema and striate keratitis, and lessen or prevent ciliary inflammatory reaction. We have seen no ill-effects from cortisone on the healing processes, especially as the sutures remain in place.

The patient is allowed to sit in a chair the same afternoon, and the first dressing is done on the second day and atropine given. If the patient is 
old, he will be out of bed at least on the two following days, and he will be up and about on the fourth day. The bandage is usually removed on the fourth or fifth day and the eye is then covered by a protective shield only. $\mathrm{He}$ is discharged on the eighth or tenth day.

Statistics show that the results achieved by Barraquer (1957) show remarkably few accidents. In 294 operations, vitreous loss occurred in only one case, and retinal detachment occurred in seven cases, but six of these were cured by lamellar resection. Long-standing post-operative keratitis occurred in one case only. In 34 cases the lens capsule ruptured, but in spite of this total extraction was obtained in eighteen of them.

During 1956, 29 cataract cases at the Finsen Institute showed few accidents. Other types of erisophake slipped off the lens once or more in ten cases out of eleven, while the Barraquer erisophake slipped only once in fifteen cases.

The development of post-operative keratitis probably depends on the delicacy of the handling of the cornea during the extraction. It is more difficult to be careful when the erisophake slips off and has to be re-inserted than when the grip is as firm as it is with the controlled vacuum of some $350 \mathrm{~mm}$. Hg. Curarization gives a deeper and more permanent anterior chamber when this is opened during the operation. This is probably the reason for the more frequent occurrence of keratitis in the cases treated at Finsen Institute, where curare has not generally been used for cataract surgery.

In all except four cases, the eventual astigmatism was less than 1 dioptre. In one case from the Finsen Institute retinal detachment followed 9 weeks after intracapsular extraction, and was cured by lamellar scleral resection of $180^{\circ}$; the eventual visual acuity was $6 / 12$.

Our experience at the Finsen Eye Department with pre-operative and operative sedation and with the controlled vacuum and special handle for the erisophake is in accordance with the statements of the Barraquer Clinic, that this method increases the safety and ease of the operation and leads to fewer post-operative complications.

\section{REFERENCES}

ArRugA, H. (1952). " Ocular Surgery”. Hoeber, New York.

AtKInson, W. S. (1955). "Anesthesia in Ophthalmology". Thomas, Springfield, Ill.

BARRAQUER BARRAQUER, IGNACIO (1917). Quoted from Kirby (1950). - (1947-8). Estud. e. inform. oftalm., vol. I, No. 1. Barcelona.

Barraquer Moner, José (1950-1). Estud. e. inform. oftalm., vol. III, No. 10. Barcelona. (1952). Estud. e. inform. oftalm., vol. IV, No. 8. Barcelona.

BARRAQUER, JoAQUIN (1954). "Anestesia potenciada en cirugia ocular". Thesis, Barcelona. (1955). Estud. e. inform. oftalm., vol. VII. Barcelona. (1957). Ibid., vol. VIII.

HARrington, D. O. (1951). Amer. J. Ophthal., 34, 435.

HuLEN, V. H. (1910). Quoted from Kirby (1950), p. 332.

KIRBY, D. B. (1950). "Surgery of Cataract". Lippincott, Philadelphia. (1955). "Advanced Surgery of Cataract". Lippincott, Philadelphia.

Malbrán, J. (1957). Amer. J. Ophthal., 44, 427.

PopPi PoPpi, MARIO (1950). Estud. e. inform. oftalm., vol. II., No. 2. Barcelona.

STOEWER, - (1902). Ber. ophthal. Ges. Heidelberg, 30, 296.

VillaseCA, A. (1956). Arch. Ophthal. (Chicago), 55, 9. 